\title{
Exploring the diversification mechanism of skeletal construction in representative natural product families
}

\author{
The Journal of Antibiotics (2016) 69, 471-472; doi:10.1038/ja.2016.69
}

\begin{abstract}
$\mathbf{T}^{\mathrm{t}}$ is our great pleasure to be Guest Editors of this special issue of 1 The Journal of Antibiotics dedicated to Professor David E Cane, upon his long-standing contribution as an editorial board member of this Journal. For over 45 years, Prof. Cane devoted much of his life to studying the biosynthesis of natural products. During this period, research techniques of biosynthetic studies have drastically advanced, as well as our understanding of nature as a synthetic chemist. Therefore, Prof. Cane's long scientific adventure itself is a rich history of natural product biosynthesis.
\end{abstract}

His research started in Prof. EJ Corey's laboratory at Harvard University (1966-1971). During his Ph.D. study, Prof. Cane developed synthetic methodologies and achieved total synthesis of trans- $\beta$ bergamotene, an unusual sesquiterpene that is found in plants and fungi. Enchanted by the beauty of nature's strategy of assembling highly complex natural products, he joined Prof. Arigoni's group as a postdoc in ETH in Switzerland (1971-1973), where he investigated sesquiterpene biosynthesis with a great deal of scientific stimulation. After he obtained an assistant professor position at Brown University, he returned to the US and began his independent academic carrier $(1973-)$, where he has been running a broad research program on natural product biosynthesis, especially those of terpenes and polyketides.

In the 1970 s to the late 1980 s, only limited analytical and genetic techniques were available; thus he adopted the application of ${ }^{13} \mathrm{C}$ NMR to the study of natural product biosynthesis through feeding experiments with isotope-labeled precursors. In terpene biosynthesis, he traced the fate of labeled atoms to speculate sequential rearrangements that occur during terpene cyclization and determined likely biogenesis pathways for compounds such as trichodiene, pentalenene, bergamotene and aristolochene. These proposals were later confirmed by enzymatic conversion experiments using cell-free systems. He greatly contributed to the understanding of stereochemical outcomes during the enzymatic transformation of the linear precursor farnesyl-diphosphate into the cyclic skeletons of terpene molecules. In polyketide biosynthesis, experiments using multiply labeled precursors $\left({ }^{2} \mathrm{H},{ }^{13} \mathrm{C}\right.$ and $\left.{ }^{18} \mathrm{O}\right)$ such as acetate/propionate and advanced intermediate analogs enabled him to propose that the macrocyclic skeletons of erythromycin and other macrolides are biosynthesized via polyhydroxy intermediates. Using a similar strategy, a unified biogenesis model of polyether antibiotics via polyenepolyepoxide intermediates was proposed.

From the 1990s to 2016, after genetic approaches to study biosynthesis became available and individual enzymes could be cloned and assayed, Prof. Cane published extensively the biochemical characterization of key biosynthetic enzymes in the pathways of terpenes and macrolide polyketides. His unparalleled chemical insights were key in unraveling the individual steps and key mechanisms behind some of the most fascinating enzymes. He also forged key collaborations with those having complementary expertise, highlighted by his collaboration with Chaitan Khosla at Stanford University (Biosynthetic Engineering), Christopher Walsh at Harvard Medical School (Mechanistic Enzymology) and David Christianson at University of Pennsylvania (Protein Crystallography).

One of the first X-ray crystal structures of terpene synthase (pentalenene synthase) was determined by Prof. Cane and his collaborators in 1997, followed by the structures of numerous other terpene synthases. These studies unveiled the universal all-helical fold of these remarkable enzymes and provided detailed pictures of how the synthetically versatile cyclases function. It was discovered that mutations in the active site produced mixtures of aberrantly cyclized products, which resulted from the redirected quenching and cyclization of enzyme-bound cationic intermediates. Analysis of these 'unnatural' products of a collection of terpene synthase mutants has provided invaluable mechanistic insights into the multistep cyclization cascades of a wide variety of terpene synthases, now widely found in textbooks. From the 2000s, after microbial genomic sequences became widely available, Prof. Cane used genome-mining approaches to functionally characterize cryptic terpene synthases of unknown functions found in Streptomyces sp. This work resulted in the isolation of many previously unknown terpene structures, led to the characterization of the corresponding terpene synthases, and elucidated the unusual pathway for well-known moist soil odor geosmin and for the highly oxygenated pentalenolactone.

One of the most complex enzyme systems for producing diverse molecular skeletons in nature is the large assembly line-like polyketide synthases (PKS). Prior to the 1990s, the biosynthetic logic of these enzymes represented one of the long-standing mysteries. In the beginning of the 1990s, the discovery of the modular organization of the PKS resulted in a giant leap and explosion of activities in PKS research. Prof. Cane, having a long-standing interest and expertise in macrolide polyketides, studied the mechanisms of 6-deoxyerythonolide B synthase (6-DEBS). In particular, his synthetic expertise allowed his group to prepare synthetic short-chain thioesters that mimic the intermediates of the PKS assembly line, and enabled him and his collaborators to interrogate the mechanistic details of 6-DEBS and other PKSs. A variety of natural and unnatural acyl chains were 
used to understand the substrate specificity and stereochemical control of individual domains, as well as the key structural and biochemical features of entire modules that determine the high-fidelity programming of complex macrocyclic polyketides. Prof. Cane recently showed that ketoreductase (KR) domains in PKSs are responsible for controlling the stereochemistry of not only the reduced $\beta$-hydroxyls but also the adjacent $\alpha$-methyl moieties during polyketide elongation. These data were further supported by the X-ray structure of KR and other domains. This finding is key to understanding how the 14-membered 6-deoxyerythronolide can amass nine stereocenters, a feature that has challenged synthetic chemists for decades.

Throughout his career, Prof. Cane has played seminal roles in advancing our understanding of the enzyme reaction mechanisms of terpene synthases and modular polyketide synthases. In recognition of these scientific achievements, he has received prestigious awards and honors such as the Prelog Lecture and Medal (ETH Zürich, 2002), Ernest Guenther Award (American Chemical Society, 1985), Arthur C. Cope Scholar Award (American Chemical Society, 2000), Alfred Bader Award (American Chemical Society, 2013) and Fellow of the American Association for the Advancement of Science (2003).

Prof. Cane has been an editorial board member of many journals and especially for The Journal of Antibiotics, for which he has served for more than 30 years (1983-). He served as a Section Editor of The Journal of Antibiotics from 2006 and was appointed as Emeritus Editorial Board member in 2014.

This special issue of The Journal of Antibiotics brings together not only a review from Prof. Cane himself, describing his personal research history with enlightening episodes, but also contributions from his colleagues, trainees and friends who have gathered to report some of their research in tribute to this remarkable mentor and colleague.

We wish to acknowledge and sincerely thank the many authors, reviewers and journal personnel whose hard work and dedicated efforts made this special issue possible.

\section{CONFLICT OF INTEREST}

The authors declare no conflict of interest.

Hideaki Oikawa ${ }^{1}$ and Yi Tang ${ }^{2}$ ${ }^{1}$ Department of Chemistry, Graduate School of Science, Hokkaido University, Hokkaido, Japan and ${ }^{2}$ Chemical and Biomolecular Engineering, University of California, Los Angeles, CA, USA E-mail: hoik@sci.hokudai.ac.jp or yitang@ucla.edu 